\section{Deep phenotyping of osteoarthritis: a step forward}

\author{
Francis Berenbaum
}

Is osteoarthritis (OA) only one and the same disease or is it actually a nebula of several diseases for which we still lack markers allowing to differentiate them? This issue is currently the subject of much debate in the medical and scientific community and remains unresolved. It is not a trivial question because deciphering disease phenotypes could be used to select a group of patients for whom a particular treatment will be effective or for whom the prognosis will be better or worse.

According to the Osteoarthritis Research Society International (OARSI), $\mathrm{OA}$ is a disorder involving movable joints characterised by cell stress and extracellular matrix degradation initiated by microinjury and macroinjury that activate maladaptive repair responses, including proinflammatory pathways of innate immunity. The disease manifests first as a molecular derangement (abnormal joint tissue metabolism), followed by anatomical and/or physiological derangements (characterised by cartilage degradation, bone remodelling, osteophyte formation, joint inflammation and loss of normal joint function), which can culminate in illness. ${ }^{1}$ It is clear that this complex definition reveals, on the one hand, a poor understanding of the underlying mechanisms, and on the other hand the potential extent of heterogeneity of the disease. With such a definition, it is easy to imagine the theoretical possibility of many different disease subgroups, depending on clinical, biological, genetics and/or imaging variables.

Some believe that the successive failures of the many therapeutic trials carried out in recent years to delay OA are due to the phenotypic heterogeneity of the patients included in these trials, masking the possible efficacy of the drug $\mathrm{X}$ reserved for the only phenotype Y. This reasoning is analogous to what is now consensually accepted in cancer, where drugs used to be poorly effective in clinical trials before

Correspondence to Dr Francis Berenbaum, Sorbonne Université, INSERM, DHU i2B, AP-HP, Hôpital SaintAntoine, Paris 75012, France;

francis.berenbaum@aphp.fr patient selection have become remarkably effective in specific phenotypes based on tumour gene signatures. ${ }^{2}$ Thus, recognising OA like a spectrum of diseases covering several phenotypes would make it possible to envisage a much more individualised medicine. It must be said that so far the vision of drug developers in this field is closer to 'one size fits all', which may explain the fact that there is no disease-modifying drugs on the market yet and that the quality of life of patients with OA remains poor.

A better knowledge of OA phenotypes could also be useful in the prognostic evaluation of the disease. Only a small percentage of patients with early knee OA will progress up to total joint space narrowing on standard radiographs even after decades. ${ }^{3}$ A better identification of fast versus slow progressors could accelerate therapeutic research by optimising the selection

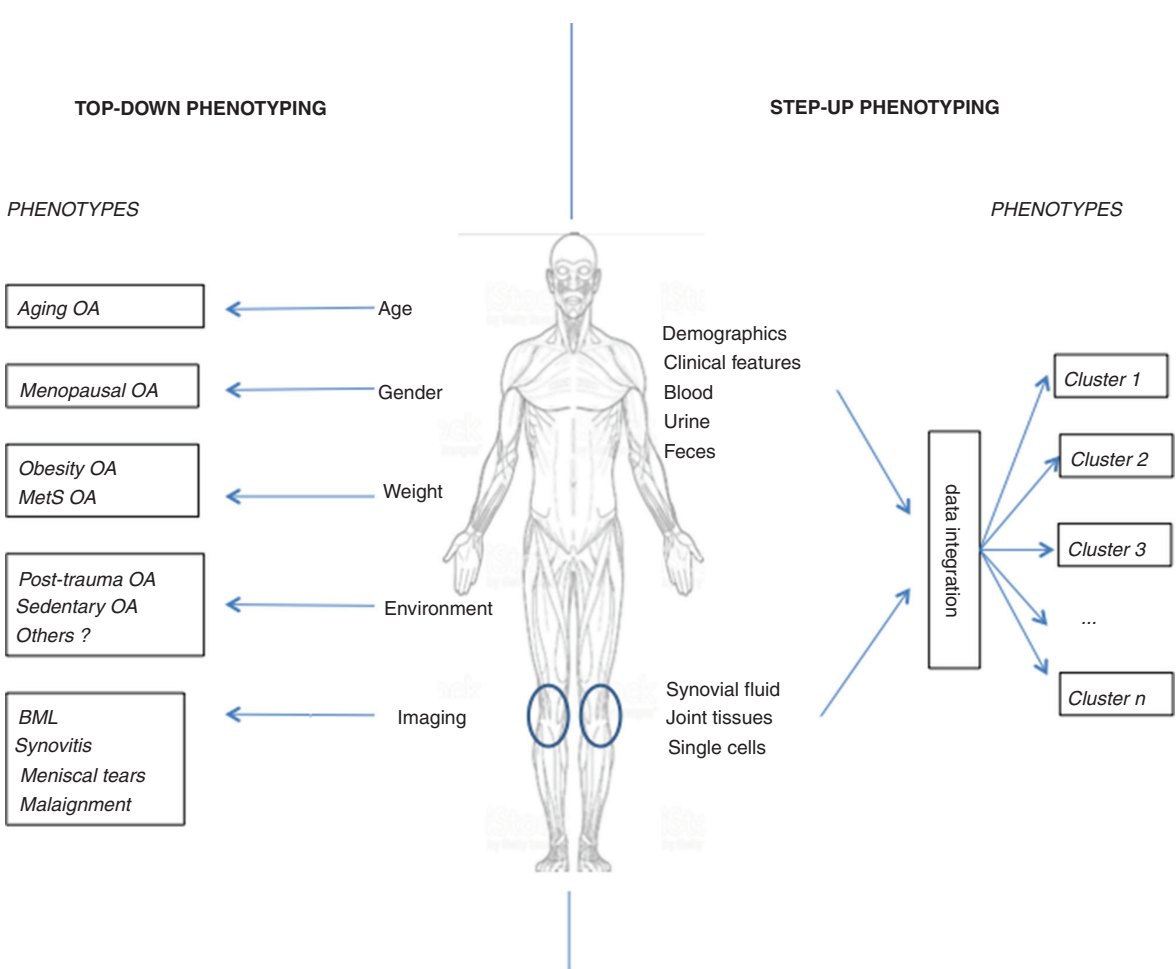

Figure 1 How to phenotype osteoarthritis? On the left side, phenotypes result from what is already known about the disease in a hypothesis-driven manner. On the right side, the phenotypes come from unsupervised clustering computational methods. The granularity of the phenotype precision depends on the number of data collected and on the quality of data integration. BML, bone marrow lesions; MetS, metabolic syndrome; OA, osteoarthritis. 
between adipokines, low-grade inflammation, metabolic syndrome and $\mathrm{OA}^{4}$ ) or to test new molecules (for instance, anti-interleukin-1 antibodies and synovitis-associated $\mathrm{OA},{ }^{5}$ bisphosphonates and bone marrow lesion-associated $\mathrm{OA}^{6}$ ). Another way to phenotype the disease, which could be described as a 'step-up' approach, is to look for these phenotypes without any a priori, relying solely on sophisticated statistical methods developed quite recently to find unsupervised clusters. ${ }^{78}$ Because of the absence of any hypothesis that drives the research, you consider that the more data you get (at the individual level) the more precise phenotypes you expect (defined as 'deep phenotyping'). The work of Ji and collaborators 9 published in Annals of the Rheumatic Diseases is a new step to a future deep-phenotyping approach in OA. It opens a very innovative, original and pioneering path in the field by taking advantage of the considerable technological progress of the last few years to study the extended expression of the transcriptome at a single cell level. The authors performed an RNA sequencing analysis on 1464 chondrocytes from 10 patients with OA undergoing total knee replacement at different stages of severity coupled to a computational analysis and histological assays of the cartilage explants. By gathering the different populations of chondrocytes according to their transcriptome, they identified seven molecularly defined clusters of chondrocytes, including three novel phenotypes with distinct functions. So far, chondrocyte subtypes were divided into proliferative chondrocytes (which are found in the proliferative zone of growth plates), prehypertrophic chondrocytes (which modulate the onset of hypertrophic chondrocytes), hypertrophic chondrocytes (which regulate the mineralisation of the surrounding cartilage matrix) and fibrocartilage chondrocytes. In this work, three unknown clusters were found. The authors called these new chondrocyte populations effector chondrocytes (enriched in genes involved in metabolism), regulatory chondrocytes (enriched in genes involved in signalling pathways) and homeostatic chondrocytes (enriched in circadian clock rhythm marker genes). It is noteworthy that there were also two subpopulations within hypertrophic chondrocytes (called HTC-A and HTC-B) which may have distinct roles in OA pathophysiology. Then, the authors investigate the relationships between these seven endotypes of OA cartilage chondrocytes and the OA severity based on a knee scoring system and the OA stage of the samples. In parallel, they assessed the expression level of 19566 protein-coding genes across the seven OA chondrocyte endotypes. By stratifying the patient samples into two groups (expression levels above or below the determined cut-off values based on each individual gene profile), they identified 336 predictive genes, with 199 related to favourable outcomes and 137 related to unfavourable outcomes. Interestingly, favourable genes were mainly expressed in the three newly described chondrocyte populations, while unfavourable genes were expressed mainly in proliferative chondrocytes, prehypertrophic chondrocytes and fibrocartilage chondrocytes. Finally, based on a correspondence analysis (a multivariate statistical technique similar to principal component analysis but applies to categorical rather than continuous data), they demonstrated that proliferative chondrocytes, prehypertrophic chondrocytes and fibrocartilage chondrocytes were correlated with worse clinical outcomes. This work sheds new light on the pathophysiology of the disease by triggering our curiosity on the lineage of these new chondrocyte phenotypes and their expression in joint development, in OA initiation and in cartilage regeneration. It also opens up our research agenda in the field of OA pathophysiology by restoring its nobility to cartilage, a tissue rather neglected in recent years for the benefit of the subchondral bone and synovial tissue.

Attempts to find molecular players in OA progression based on -omics technologies have already been published. The study published in this issue is the first one that looks at such molecular players at the single cell level, and also that delineates how the repartition of different cell populations could discriminate patient phenotypes according to clinical outcomes. There are, however, a number of limitations to this study, especially if the ultimate goal is to improve the management of patients. First, this study is based on a limited number of samples since only 10 patients were the subject of this experimental study. It cannot be held to the authors because the current technology makes it challenging to perform such studies on hundreds of samples. There is no doubt that the rapid progress in big data management, bioinformatics and molecular biology technologies should solve this problem quickly. Second, it is obvious that in real life such a phenotypic approach based on the molecular analysis of cartilage cells makes it almost impossible today to apply in current practice because of the aggressiveness of the procedure in obtaining cartilage, even if cartilage samples are already being taken today to treat traumatic cartilage defects in non-bearing areas. ${ }^{10}$ Third, we lack longitudinal data to know whether these chondrocyte phenotypes are fixed or whether there may be a change from one phenotype to another over time according to OA progression. Moreover, cells present in the other joint tissues such as synoviocytes, osteoblasts and osteoclasts (to name only three) could also play critical roles in the outcome, justifying to realise this same kind of analysis in other joint tissues in the future.

Altogether, this work shows us brilliantly how new technologies in the molecular biology field coupled with the considerable progress of these last years in bioinformatics can help us to better understand the pathophysiology of the disease, but also how concretely we can hope to improve the prognosis and the treatment. The new challenge in the next years will be to integrate these huge amounts of clinical, biological, genetics, imaging and -omics data sets in order to eventually delineate useful phenotypes for predicting outcomes and treatment responses (figure 1). This new step of data integration for future deep phenotyping is just starting in our field, ${ }^{11}$ and the tools for integrating these numerous layers of data sets should be operational very soon. Precision medicine can now be reasonably considered as an achievable option in $\mathrm{OA}$ in the next decade.

Handling editor Josef S Smolen

Contributors $\mathrm{FB}$ : design and writing.

Funding FB is supported by ROAD-Fondation Arthritis Network and Société Française de Rhumatologie.

Competing interests None declared.

Patient consent Not required.

Provenance and peer review Commissioned; internally peer reviewed.

(c) Author(s) (or their employer(s)) 2019. No commercial re-use. See rights and permissions. Published by BMJ.

Check for updates

To cite Berenbaum F. Ann Rheum Dis 2019;78:3-5.

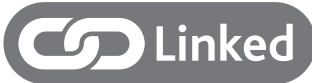

- http://dx.doi.org/10.1136/annrheumdis-2017212863

Ann Rheum Dis 2019;78:3-5.

doi:10.1136/annrheumdis-2018-213864

\section{REFERENCES}

1 Kraus VB, Blanco FJ, Englund $M$, et al. Call for standardized definitions of osteoarthritis and risk 
stratification for clinical trials and clinical use. Osteoarthritis Cartilage 2015;23:1233-41.

2 Nevins JR, Potti A. Mining gene expression profiles: expression signatures as cancer phenotypes. Nat Rev Genet 2007;8:601-9

3 Leyland KM, Hart DJ, Javaid MK, et al. The natural history of radiographic knee osteoarthritis: a fourteenyear population-based cohort study. Arthritis Rheum 2012:64:2243-51.

4 Berenbaum F. Osteoarthritis as an inflammatory disease (osteoarthritis is not osteoarthrosis!). Osteoarthritis Cartilage 2013;21:16-21.

5 Fleischmann R, Bliddal H, Blanco $\mathrm{F}$, et al. SAT0575 Safety and efficacy of lutikizumab (ABT981), an anti-interleukin-1 alpha/beta dual variable domain (DVD) immunoglobulin, in subjects with knee osteoarthritis: results from the randomised, doubleblind, placebo-controlled, parallel-group phase 2 trial. Ann Rheum Dis 2018:77:1141-2.

6 Laslett LL, Doré DA, Quinn SJ, et al. Zoledronic acid reduces knee pain and bone marrow lesions over 1 year: a randomised controlled trial. Ann Rheum Dis 2012;71:1322-8.

7 van der Esch M, Knoop J, van der Leeden $\mathrm{M}_{\text {, }}$ et al. Clinical phenotypes in patients with knee osteoarthritis: a study in the Amsterdam osteoarthritis cohort. Osteoarthritis Cartilage 2015:23:544-9.

8 Knoop J, van der Leeden M, Thorstensson CA, et al. Identification of phenotypes with different clinical outcomes in knee osteoarthritis: data from the Osteoarthritis Initiative. Arthritis Care Res 2011;63:1535-42.

9 Ji Q, Zheng Y, Zhang G, et al. Single-cell RNAseq analysis reveals the progression of human osteoarthritis. Ann Rheum Dis 2019:78:100-10.

10 Brittberg M, Lindahl A, Nilsson A, et al. Treatment of deep cartilage defects in the knee with autologous chondrocyte transplantation. N Eng/ J Med 1994:331:889-95.

11 Steinberg J, Ritchie GRS, Roumeliotis TI, et al. Integrative epigenomics, transcriptomics and proteomics of patient chondrocytes reveal genes and pathways involved in osteoarthritis. Sci Rep 2017;7:8935 